\title{
Effect of vitamin D on the recurrence rate of rheumatoid arthritis
}

\author{
JUNXIA YANG ${ }^{1}$, LIN LIU $^{1}$, QINGLIN ZHANG ${ }^{2}$, MEIRONG LI $^{1}$ and JINGYA WANG ${ }^{1}$ \\ ${ }^{1}$ Department of Rheumatology, ${ }^{2}$ Central Laboratory, Xuzhou Central Hospital, Xuzhou, Jiangsu 221000, P.R. China
}

Received July 22, 2015; Accepted September 8, 2015

DOI: $10.3892 /$ etm.2015.2747

\begin{abstract}
Rheumatoid arthritis (RA) is an autoimmune disease that is characterized by chronic inflammation affecting target tissues including the joints, bones, and synovial membrane. However, the etiology and pathogenesis of RA remains to be determined, and investigations into the treatment of RA are imperative. Vitamin D (Vit D) was previously found to be associated with the activity of RA and exerts therapeutic benefits. The aim of the present study was to investigate the effect of Vit D on the recurrence rate of RA. A total of 377 patients with RA at remission stage were divided into the normal Vit D group and the Vit D-deficient group according to their Vit D levels at baseline. The patients in the Vit D-deficient group were further randomly allocated to receive treatment with or without Vit D. The patients were followed up for 24 months, and the visual analogue scale, as well as the number of pain and swelling joints were recorded every 2-3 months. In addition, C-reactive protein and the blood sedimentation rate were measured every 2-3 months to assess the recurrence of RA based on disease activity score 28 (DAS28). The recurrence rate of RA was 16.7, 19.0 and $29.5 \%$ for the normal Vit D group $(n=168)$, Vit $D$ treatment subgroup $(n=84)$, and non-Vit $D$ treatment subgroup $(n=88)$, respectively. The recurrence rate of the normal Vit D group was lower, compared to the non-Vit D treatment subgroup, and the difference was statistically significant. However, the difference was not statistically significant between the Vit D treatment subgroup and non-Vit D treatment subgroup. In conclusion, a decreased level of Vit D is a risk factor for the recurrence of RA.
\end{abstract}

\section{Introduction}

Rheumatoid arthritis (RA) is an autoimmune disease characterized by chronic inflammation affecting target tissues including the joints, bones, and synovial membrane. RA

Correspondence to: Dr Lin Liu, Department of Rheumatology, Xuzhou Central Hospital, 199 Jiefang South Road, Xuzhou, Jiangsu 221000, P.R. China

E-mail: yangjunxia0127@126.com

Key words: recurrence, arthritis, rheumatoid, vitamin D, randomized controlled trial causes a high rate of disability and ultimately leads to death in patients (1). However, the etiology and pathogenesis of RA have yet to be determined. Thus, investigations into treatment strategies for RA are crucial. Currently, most studies are based on the premise that RA develops through an antigentriggered 'challenge-chained immune reaction' process, with infection and autoimmune responses playing central roles in the development and progression of RA, while genetic and environmental factors are important influencing factors that may activate Th1 cells and increase the secretion of cytokines including interleukin-1 (IL-1), IL-6 and TNF- $\alpha$ through the major histocompatibility complex $(2,3)$. These cytokines are useful in mediating the continuous activation of B cells and eventually induce synovial lesions $(2,3)$.

Vitamin D (Vit D) decreases the production of IL-17, IL-6, IL-1 and tumor necrosis factor- $\alpha(\mathrm{TNF}-\alpha)$ by inhibiting the immune response of Th1 cells. In addition, activated Vit D may inhibit the precursors of monocytes by immune modulation effects and inhibit the apoptosis of B-cells (4). It has been previously demonstrated that Vit D is associated with the activity of RA and exerts therapeutic benefits $(5,6)$. Although in previous studies the association between Vit D and RA was investigated, to the best of our knowledge, no study has investigated whether Vit D affects the recurrence rate of RA or whether Vit D supplementation has various benefits for RA patients through reduction of the recurrence of RA, thereby proving useful for the long-term remission of RA, or reducing the application of slow-acting drugs. In this randomized, open-label clinical study, we investigated the effect of Vit D on the recurrence rate of RA and found that a decreased level of Vit D in the groups examined in the present study is a risk factor for the recurrence rate of RA.

\section{Materials and methods}

Patients characteristics. RA patients, who were treated at the outpatient service of the Department of Rheumatism and Immunology, Xuzhou Central Hospital (Jiangsu, China), between October 2010 and February 2014, were enrolled. A questionnaire was used to collect general data including age, gender, disease duration, and medical history in the previous 2-3 months, visual analogue scale (VAS) score, patient global assessment and number of swelling and pain joints of the patients. In addition, levels of C-reactive protein (CRP), blood sedimentation rate (ESR), Ca, $\mathrm{P}$ and $25(\mathrm{OH}) \mathrm{D} 3$ of patients were also measured. The inclusion criteria that applied were: i) patients met the American College of Rheumatology and European League Against Rheumatism (ACR-EULAR) 
Table I. Demographic data and baseline disease characteristics.

Vit D-deficient group

\begin{tabular}{lcccc}
\cline { 3 - 4 } Characteristics & $\begin{array}{c}\text { Normal Vit } \mathrm{D} \\
\text { group }(\mathrm{n}=168)\end{array}$ & $\begin{array}{c}\text { Vit } \mathrm{D} \text { treatment } \\
\text { subgroup }(\mathrm{n}=84)\end{array}$ & $\begin{array}{c}\text { Control subgroup } \\
(\mathrm{n}=88)\end{array}$ & P-value \\
\hline Age (years) & $43.1 \pm 7.1$ & $44.2 \pm 7.5$ & $41.7 \pm 8.6$ & 0.32 \\
Male patients, $\mathrm{n}(\%)$ & $20(11.9)$ & $12(14.2)$ & $10(11.3)$ & 0.82 \\
Disease duration (years) & $5.2 \pm 2.4$ & $4.9 \pm 2.1$ & $5.1 \pm 2.2$ & 0.78 \\
Remission time (months) & $8.2 \pm 4.1$ & $7.9 \pm 4.6$ & $8.6 \pm 3.8$ & 0.73 \\
Vit D level (ng/ml) & $37.6 \pm 7.1$ & $20.6 \pm 9.1$ & $21.3 \pm 8.5$ & $0.71^{\text {a }}$ \\
\hline
\end{tabular}

${ }^{\mathrm{a} C}$ Comparison of the Vit D level between the Vit D treatment and non-Vit D subgroups. Vit D, vitamin D.

classification criteria for RA issued in 2010 (7); ii) patient was in a remission phase in the previous $2-3$ months, according to the ACR-EULAR 2010 criteria (8), with $<1$ swelling joint and 1 tender joint, CRP $<1 \mathrm{mg} / \mathrm{dl}$, and patient global assessment $\leq 1$ (range $0-10$ ); and iii) patient did not receive glucocorticoids or Vit $\mathrm{D}$ during the previous 2-3 months, although the patients remained on maintenance treatments with slow-acting drugs. Patients with one or more of the following features were excluded: i) other comorbid rheumatic diseases; ii) poor compliance to previously used treatment method; or iii) serum calcium $>2.75 \mathrm{mmol} / 1$ or serum phosphorus $>1.61 \mathrm{mmol} / 1$.

The study was approved by the Ethics Committee of the Clinical Trial Center of the Xuzhou Center Hospital (20101016-10). Written informed consent was obtained from all the patients prior to entering the study.

Treatment method. This randomized, controlled, openlabel study was conducted between October 2010 and February 2014. The included RA patients were first divided into the normal Vit D and Vit D-deficient groups according to their baseline Vit D levels (cut-off value, $30 \mathrm{ng} / \mathrm{dl}$ ). Random numbers were generated by a computer to allocate patients in the Vit D-deficient group, the Vit D treatment subgroup and the non-Vit D treatment subgroup (control group). For the patients in the Vit D treatment subgroup, alfacalcidol $(0.25 \mu \mathrm{g}$, twice a day) was administered as the treatment, while for patients in the control group and non-Vit D treatment subgroup the previously used treatment method was continued (Table II). Patients were followed up every 2-3 months during the 24-month follow-up period, and VAS and the number of swelling or tender joints were recorded. In addition, CRP and ESR levels were measured. The activity level was calculated based on the disease activity score 28 (DAS28). When DAS28 $\geq 3.2$ it was deemed as RA recurrence. The Vit D3 level of the patients was also measured at the end of follow-up. In addition, $\mathrm{Ca}$ and $\mathrm{P}$ levels were measured for the patients who received alfacalcidol. If patients receiving alfacalcidol were found with a serum calcium level $>2.75 \mathrm{mmol} / 1$ or serum phosphorus $>1.61 \mathrm{mmol} / 1$, treatment of alfacalcidol was discontinued and additional treatments were performed to reduce serum calcium and phosphorus. Enzyme-linked immunosorbent assay (ELISA) kits (Roche,
Basel, Switzerland) were used to determine Vit D3 levels, and blood samples were preserved in the dark.

Statistical analysis. SPSS 16.0 software (SPSS, Inc., Chicago, IL, USA) was used for statistical analyses. Analysis of variance and the t-test (two-sample/group t-test and least significant difference test) were used for comparison of quantitative data. The Chi-square test was used for comparison of qualitative data. $\mathrm{P}<0.05$ was considered to indicate a statistically significant result.

\section{Results}

General characteristics. A total of 377 eligible patients were included in the present study, of which 185 patients had normal Vit D levels and 192 patients had Vit D deficiency, which indicated a Vit D deficiency rate of $50.9 \%$. The 192 patients with Vit D deficiency were subdivided into the Vit D treatment subgroup $(n=93$, comprising 77 women and 16 men) and the non-Vit D treatment subgroup $(n=99$, comprising 83 women and 16 men). Table I shows the demographic and clinical characteristics of the patients at baseline. No significant difference in age, gender, disease duration, and remission time at study entry was identified for the three groups. In addition, the Vit D level was not significantly different between the Vit D treatment and non-Vit D treatment subgroups. Table II provides the treatment methods applied at study entry for the patients, and the data show there was no statistically significant difference. Vit D levels at baseline were significantly different between the recurrent patients in the Vit D treatment and non-Vit D treatment subgroups (Table III).

Recurrence rate. After 24 months of follow up, the number of patients lost to follow up and who failed to comply with treatment requirements in the normal Vit D group, Vit D treatment subgroup and control subgroup was 17, 9 and 11, respectively. Thus the actual number for the three groups of complete follow up was 168 (comprising 149 women and 19 men), 84 (comprising 72 women and 12 men), and 88 (comprising 78 women and 10 men) for the the normal Vit D group, Vit D treatment subgroup and non-Vit D subgroup, respectively. 
Table II. Treatment methods of patients at study entry.

\begin{tabular}{lccc}
\hline & & \multicolumn{2}{c}{ Vit D-deficient group } \\
\cline { 3 - 4 } Treatment & $\begin{array}{c}\text { Normal Vit D } \\
\text { group }(\mathrm{n}=168)\end{array}$ & $\begin{array}{c}\text { Vit D treatment } \\
\text { subgroup }(\mathrm{n}=84)\end{array}$ & $\begin{array}{c}\text { Control subgroup } \\
(\mathrm{n}=88)\end{array}$ \\
\hline Methotrexate & 30 & 16 & 16 \\
Leflunomide & 22 & 12 & 10 \\
Tripterygium and methotrexate & 16 & 10 & 8 \\
Methotrexate and hydroxychloroquine & 60 & 28 & 32 \\
Leflunomide and methotrexate & 40 & 18 & 22 \\
\hline
\end{tabular}

${ }^{\text {aP }}>0.05$, comparing each treatment method for the three groups. Vit D, vitamin D.

Table III. Comparison of the general characteristics of the patients with recurrence.

\begin{tabular}{lccc}
\hline & & \multicolumn{2}{c}{ Vit D-deficient group } \\
\cline { 3 - 4 } Characteristics & $\begin{array}{c}\text { Normal Vit D } \\
\text { group (n=168) }\end{array}$ & $\begin{array}{c}\text { Vit D treatment } \\
\text { subgroup (n=84) }\end{array}$ & $\begin{array}{c}\text { Control subgroup } \\
(\mathrm{n}=88)\end{array}$ \\
\hline Age (year) & $42.1 \pm 6.2$ & $41.2 \pm 6.6$ & $41.7 \pm 5.8$ \\
Disease duration (years) & $5.0 \pm 1.9$ & $4.9 \pm 1.7$ & $5.1 \pm 2.1$ \\
Remission time (months) & $8.1 \pm 3.9$ & $7.9 \pm 4.1$ & $8.2 \pm 3.7$ \\
Vitamin D level (ng/ml) & $36.8 \pm 6.7$ & $25.5 \pm 8.9$ & $20.9 \pm 8.6$ \\
\hline
\end{tabular}

${ }^{a}$ Comparison of Vit D level between patients with disease recurrence in the Vit D treatment and non-Vit D subgroups. Vit D, vitamin D.

Table IV. Comparison of recurrence rate for the three groups.

\begin{tabular}{|c|c|c|c|c|c|}
\hline \multirow[b]{2}{*}{ Group } & \multirow[b]{2}{*}{$\begin{array}{l}\text { No. of patients } \\
\text { with recurrence }\end{array}$} & \multirow[b]{2}{*}{$\begin{array}{c}\text { No. of patient } \\
\text { without } \\
\text { recurrence }\end{array}$} & \multicolumn{3}{|c|}{$\mathrm{P}$-value } \\
\hline & & & $\begin{array}{l}\text { Comparison } \\
\text { between } \\
\text { three groups }\end{array}$ & $\begin{array}{l}\text { Comparison between } \\
\text { Vit D treatment } \\
\text { subgroup and } \\
\text { non-Vit D subgroup }\end{array}$ & $\begin{array}{c}\text { Comparison } \\
\text { between normal } \\
\text { Vit D group and } \\
\text { non-Vit D subgroup }\end{array}$ \\
\hline $\begin{array}{l}\text { Normal Vit D group } \\
(n=168)\end{array}$ & 28 & 140 & 0.05 & 0.11 & 0.02 \\
\hline Vit D-deficient group & & & & & \\
\hline $\begin{array}{l}\text { Vit D treatment } \\
\text { subgroup }(n=84)\end{array}$ & 16 & 68 & & & \\
\hline $\begin{array}{l}\text { Non-Vit D subgroup } \\
(\mathrm{n}=88)\end{array}$ & 26 & 62 & & & \\
\hline
\end{tabular}

Vit D, vitamin D.

RA recurrence was identified in 28 of the 168 patients with normal Vit D (recurrence rate of $16.7 \%$ ) compared with 26 patients in the non-Vit D treatment subgroup (recurrence rate of $29.5 \%$ ), and the difference was statistically significant $(\mathrm{P}=0.02)$. No patient in the Vit $\mathrm{D}$ treatment subgroup discon- tinued because of high calcium or high phosphorus. However, 16 patients in the Vit D treatment subgroup were identified as having RA recurrence, yielding a recurrence rate of $19.0 \%$, which was not statistically different from the recurrence rate in the non-Vit D treatment subgroup (29.5\%) $(\mathrm{P}=0.11)$ (Table IV). 


\section{Discussion}

Previous studies have demonstrated that Vit D supplementation reduces the prevalence and activity of RA (5,9-11). However, few studies have investigated the effects of Vit D on the recurrence rate of RA. In this randomized, open-label clinical study, remission phase RA patients with decreased Vit D levels were treated with or without Vit $\mathrm{D}$, and the recurrence rate of RA was compared. The results showed that the recurrence rate was statistically different between the normal Vit D and Vit D-deficient groups. The results suggest that a low Vit D level constituted a risk factor for RA recurrence. Treatment with alfacalcidol increased the Vit D levels in patients; however, the recurrence rate was not statistically different between the Vit D treatment and non-Vit D treatment subgroups.

RA is an autoimmune disorder with a complex pathophysiology. Genetic and environmental factors contribute to the development of RA. 1,25-Dihydroxyvitamin D3 has been hypothesized to decrease chondrolysis by the IL-1A-mediated production of matrix metalloproteinases, and thus, deficiency is a potential trigger for cartilage damage in RA (9). Epidemiological studies have demonstrated that a reduced Vit D level was associated with an increased risk for the development and progression of autoimmune diseases including RA, systemic lupus erythematosus, and type 1 diabetes (10-12). Increased intake of Vit D has also been associated with a reduced risk of developing RA. In a study by Merlino et al, data from 29,368 women aged between 55 and 69 years were analyzed (13). Following administration of Vit D administered in these women for 11 years, no RA was found, suggesting that Vit D reduces the incidence or RA. Merlino et al reported an inverse association between Vit D levels and the risk of RA (13). In basal pretreatment conditions, a proportional inverse relationship among the levels of 25(OH)D and the tender joint counts, HAQ scores, CRP levels, and the DAS28 scores was observed. Each increase of $10 \mathrm{ng} / \mathrm{ml}$ in the level of $25(\mathrm{OH}) \mathrm{D}$ was associated with a decrease in the DAS28 score of 0.3 and in the CRP level of $25 \%$ (14). Kröger et al studied 143 Finnish women with RA and found that the lowest values of serum Vit D were identified in patients with the highest disease activity (15). In a study with 19 patients with RA treated with traditional disease-modifying anti rheumatic drugs (DMARDs), oral supplementation with high doses of alfacalcidol for 3 months reduced the severity of the symptoms in $89 \%$ of the patients, $45 \%$ of whom achieved complete remission, and $44 \%$ had satisfactory results. No significant adverse effects were identified, and the serum calcium levels were within normal limits for all the patients during the period of the study (16).

Vit D, closely associated with RA, is cost-effective with few side effects. Vit D is recommended for the preventive treatment for high-risk individuals with RA (17). Therefore, a lack of Vit D treatment recommendations for patients with RA in remission is due to the lack of studies available on the effect of $\mathrm{Vit} \mathrm{D}$ on the RA recurrence rate. A single study focusin on Vit D and the recurrence rate of RA was performed in Iran, between October 2012 and February 2013, with a total of 80 RA patients with Vit D deficiency being enrolled and randomly allocated to receive Vit D or placebo in the 6-month follow-up period. The study showed that the flare rate was not different between two groups (18). The main drawbacks of this study were the small sample size, lack of controls with RA patients of normal Vit D levels, and the shorter observation time period of 6 months.

In the present study, the effect of Vit D on the recurrence rate of RA was evaluated in 340 RA patients in remission after a follow-up period of 24 months. Based on this study, $50.9 \%$ of RA patients in remission had Vit D deficiency. This finding was consistent with findings by Dehghan et al (18).

The results of the present study have shown that the recurrence rate was $16.7,19.0$ and $29.5 \%$ for patients in the normal Vit D group, Vit D treatment subgroup, and non-Vit D treatment subgroup. The recurrence rate of the normal Vit D group was lower, compared with that of the non-Vit D treatment subgroup, and the difference was statistically significant, suggesting that a decreased level of Vit D is a risk factor for RA recurrence. The findings of the present study demonstrate that the recurrence rate of the normal Vit D group was lower and treatment of patients with a standard dose of Vit D potentially increased the level of Vit D and reduced the recurrence rate of RA, although the difference was not statistically significant. This finding may be due to the fact that the Vit D level of the Vit D treatment subgroup following treatment remained below the normal range. Therefore, more studies are required to investigate whether increasing the dose of Vit D is beneficial to patients in order to recover normal Vit D levels, and thus affect the recurrence rate of RA. Vit D may exert important effects on autoimmune diseases, and the adverse effects of Vit D are substantially lower than slow-acting drugs.

\section{References}

1. Pinals RS: Survival in rheumatoid arthritis. Arthritis Rheum 30: 473-475, 1987.

2. Turhanoglu AD, Guler H, Yonden Z, Aslan F, Mansuroglu A and Ozer C: The relationship between vitamin $\mathrm{D}$ and disease activity and functional health status in RA. Rheumatol Int 31: 911-914, 2011.

3. Prietl B, Treiber G, Pieber TR and Amrein K: Vitamin D and immune function. Nutrients 5: 2502-2521, 2013.

4. Chen S, Sims GP, Chen XX, Gu YY, Chen S and Lipsky PE: Modulatory effects of 1,25-dihydroxyvitamin D3 on human B cell differentiation. J Immunol 179: 1634-1647, 2007.

5. Craig SM, Yu F, Curtis JR, Alarcón GS, Conn DL, Jonas B, Callahan LF, Smith EA, Moreland LW, Bridges SL Jr, et al: Vitamin D status and its associations with disease activity and severity in African Americans with recent-onset RA. J Rheumatol 37: 275-281, 2010

6. Wang M, Chen D, Lou Y and Wan R: Association study between 25(OH) vitamin D and early RA. Lab Med Clin 7: 1076-1077, 2010.

7. Aletaha D, Neogi T, Silman AJ, Funovits J, Felson DT, Bingham CO III, Birnbaum NS, Burmester GR, Bykerk VP, Cohen MD, et al: 2010 rheumatoid arthritis classification criteria: an American College of Rheumatology/European League Against Rheumatism collaborative initiative. Ann Rheum Dis 69: 1580-1588, 2010.

8. Haque UJ and Bartlett SJ: Relationships among vitamin D, disease activity, pain and disability in RA. Clin Exp Rheumatol 28: 745-747, 2010.

9. Tetlow LC and Woolley DE: The effects of 1 alpha,25-dihydroxyvitamin $\mathrm{D}(3)$ on matrix metalloproteinase and prostaglandin $\mathrm{E}(2)$ production by cells of the rheumatoid lesion. Arthritis Res 1: 63-70, 1999.

10. Cutolo M, Otsa K, Laas K, Yprus M, Lehtme R, Secchi ME, Sulli A, Paolino S and Seriolo B: Circannual vitamin D serum levels and disease activity in RA: Northern versus Southern Europe. Clin Exp Rheumatol 24: 702-704, 2006.

11. May E, Asadullah K and Zügel U: Immunoregulation through 1,25-dihydroxyvitamin D3 and its analogs. Curr Drug Targets Inflamm Allergy 3: 377-393, 2004. 
12. Andjelkovic Z, Vojinovic J, Pejnovic N, Popovic M, Dujic A, Mitrovic D, Pavlica L and Stefanovic D: Disease modifying and immunomodulatory effects of high dose 1 alpha $(\mathrm{OH}) \mathrm{D} 3$ in RA patients. Clin Exp Rheumatol 17: 453-456, 1999.

13. Merlino LA, Curtis J, Mikuls TR, Cerhan JR, Criswell LA and Saag KG; Iowa Women's Health Study: Vitamin D intake is inversely associated with RA: results from the Iowa Women's Health Study. Arthritis Rheum 50: 72-77, 2004.

14. Patel S, Farragher T, Berry J, Bunn D, Silman A and Symmons D: Association between serum vitamin D metabolite levels and disease activity in patients with early inflammatory polyarthritis. Arthritis Rheum 56: 2143-2149, 2007.

15. Kröger H, Penttilä IM and Alhava EM: Low serum vitamin D metabolites in women with RA. Scand J Rheumatol 22: 172-177, 1993.
16. Andjelkovic Z, Vojinovic J, Pejnovic N, Popovic M, Dujic A, Mitrovic D, Pavlica L and Stefanovic D: Disease modifying and immunomodulatory effects of high dose $1 \mathrm{a}(\mathrm{OH}) \mathrm{D} 3$ in RA patients. Clin Exp Rheumatol 17: 453-456, 1999.

17. Harel M and Shoenfeld Y: Predicting and preventing autoimmunity, myth or reality? Ann NY Acad Sci 1069: 322-345, 2006

18. Dehghan A, Rahimpour S, Soleymani-Salehabadi H and Owlia MB: Role of vitamin D in flare ups of rheumatoid arthritis. Z Rheumatol 73: 461-464, 2014. 